\title{
The new method of investigating the orientation of galaxies and their clusters.
}

\author{
Włodzimierz Godłowski ${ }^{1 *}$, Paulina Pajowska ${ }^{1}$, Piotr Flin $^{2}$ and \\ Elena Panko ${ }^{3}$ \\ ${ }^{1}$ Institute of Physics, Opole University, Opole, Poland \\ ${ }^{2}$ Institute of Physics, Jan Kochanowski University, Kielce, Poland \\ ${ }^{3}$ Kalinenkov Observatory, Nikolaev State University, Nikolaev, Ukraine
}

\begin{abstract}
We present a new method of analyzing galaxy alignments in clusters originaly proposed by Godowski 2012 and now improved.
\end{abstract}

Keywords. galaxies:

\section{Introduction}

Problem of large structure formation is one of the most important problems in modern cosmology and extragalactic astronomy. Classical theories (Peebles 1969; Zeldovich 1970, Efstathiou \& Silk 1983) were developed and modified by various researchers (e.g. Lee \& Penn 2002; Navarro, Abadi \& Steinmetz 2004, Codis et al. 2012, Varela et al. 2012). Various scenarios of large scale structure formation make different predictions concerning orientation of galaxies in structures, distribution of spins of galaxies and alignment between the brightest galaxy and the major axis of the structure. The ultimate test for a given scenerio would be to test it against observations. A new method of analyzing galaxy alignments in clusters proposed by Godłowski (2012) has now been improved. The distribution of position angles for galaxy major axes has been analyzed, as well as the distribution of the two angles describing the spatial orientation of the galaxy plane, both of which provide information about galaxy angular momenta.

\section{Aim}

The aim of the project is to investigate the correlation function of orientation of galaxies in clusters and the orientation of clusters themselves.

\section{References}

Codis, S., Pichon, C., Devriendt, J., Slyz, A., et al. 2012 Mon. Not. R. Astr. Soc., 427, 3320

Efstathiou, G. A. \& Silk, J., 1983, The Formation of Galaxies, Fundamentals of Cosm. Phys. 9, 1

Godłowski, W. 2012, ApJ747, 7

Lee, J. \& Pen, U. 2002, ApJ, 567, L111

Navarro, J. F., Abadi, M. G., Steinmetz, M., 2004 Astrophys. J. 613, L41

Peebles, P. J. E., 1969 Astrophys. J. 155, 393

Varela, J., Betancort-Rijo, J., Trujillo, I., \& Ricciardelli, E. 2012, Astrophys. J. 744, 82

Zeldovich, B. Ya. 1970, $A \mathscr{E} A, 5,84$ 